\title{
Paracentral acute middle maculopathy in non- ischaemic central retinal vein occlusion: the role of en face optical coherence tomography
}

\author{
Fares Antaki ㄷ, , Daniel Milad, ${ }^{1}$ Jean-Yves Sahyoun, ${ }^{1}$ Razek Georges Coussa ${ }^{2}$
}

${ }^{1}$ Department of Ophthalmology,

Centre Hospitalier de

I'Université de Montréal

(CHUM), Montreal, Quebec,

Canada

${ }^{2}$ Department of Ophthalmology and Visual Sciences, The

University of lowa Hospitals and

Clinics, lowa City, lowa, USA

\section{Correspondence to}

Dr Fares Antaki;

fares.antaki@umontreal.ca

Accepted 26 October 2021

\section{DESCRIPTION}

A 64-year-old man presented with an acute-onset central visual deficit in the left eye. He described a specific pattern of paracentral scotoma consisting of 'grey spots arranged in a honeycomb pattern'. He was a 10 pack-year smoker and his medications included low-dose aspirin, which was prescribed, 2 years prior, following an episode of amaurosis fugax in the same eye. Investigations at that time had not revealed significant carotid stenosis. He was also known for ocular hypertension secondary to pigment dispersion syndrome and had stopped adhering to his prescribed daily timolol $0.5 \%$ 3 months prior to presentation.

Spectacle-corrected visual acuity was 20/100 in the left eye (baseline, 20/20) and intraocular pressure was $28 \mathrm{~mm} \mathrm{Hg}$. Fundus examination showed central retinal vein occlusion (CRVO) (figure 1A) and revealed patchy areas of deep retinal whitening at the macula (figure 1B). Spectral domain optical coherence tomography (OCT) obtained through the zones of retinal whitening was consistent with paracentral acute middle maculopathy (PAMM) (figure 2A). The corresponding mid-retina en face OCT revealed a fern-like pattern of perivenular hyperreflectivity (figure 2B). The patient was diagnosed with non-ischaemic CRVO (based on fluorescein angiography, images not shown), with concurrent PAMM. At 2 months follow-up, visual acuity remained stable and the paracentral scotoma persisted. Follow-up imaging showed attenuation of the inner nuclear layer (INL) (figure 2C) and regression of the PAMM lesions (figure 2D).

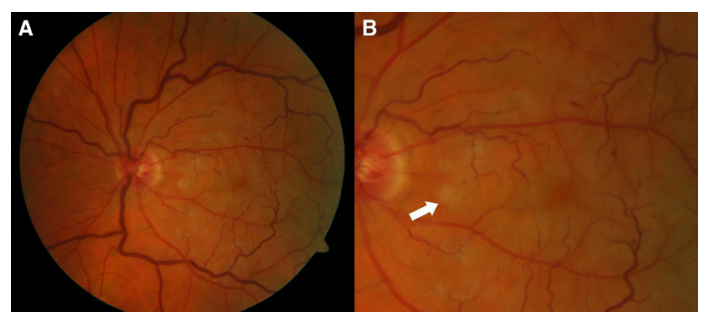

Figure 1 Colour fundus photograph of the left eye (A) showing tortuosity and dilation of the central retinal vein, mild dot-and-blot haemorrhages and optic disc hyperaemia, consistent with central retinal vein occlusion. A magnified image of the macula (B) shows multiple patchy areas of deep retinal whitening. The lesions appear dull grey-white in colour and deep in the retina (white arrow).
PAMM is an OCT finding consisting of characteristic hyperreflective band-like lesions involving the INL that can complicate up to $5.2 \%$ of nonischaemic CRVOs. ${ }^{1}$ These lesions develop in response to ischaemia of the deep retinal capillary plexuses (DCP). ${ }^{2}$ The sudden occlusion of the central retinal vein causes a marked increase in the intraluminal pressure across the entire retinal capillary bed. This in turn leads to ischaemia in regions of vascular instability like the watershed zones of the outer plexiform layer (OPL) and INL where oxygen is supplied by both the choroidal and retinal circulations. ${ }^{1}$ En face OCT allows the identification of three PAMM patterns: arteriolar, globular and fern-like pattern.$^{3}$ The fern-like pattern, which is seen in patients with CRVO, as in our case, seems to be due to the high density of capillaries, including deep plexus ones, in the perivenular distribution. ${ }^{45}$

In non-ischaemic CRVO, subjective reports of paracentral scotomas may be overlooked and identifying a perivenular fern-like pattern of PAMM is clinically significant. It can also aid the diagnosis of non-ischaemic CRVO in cases with minimal retina

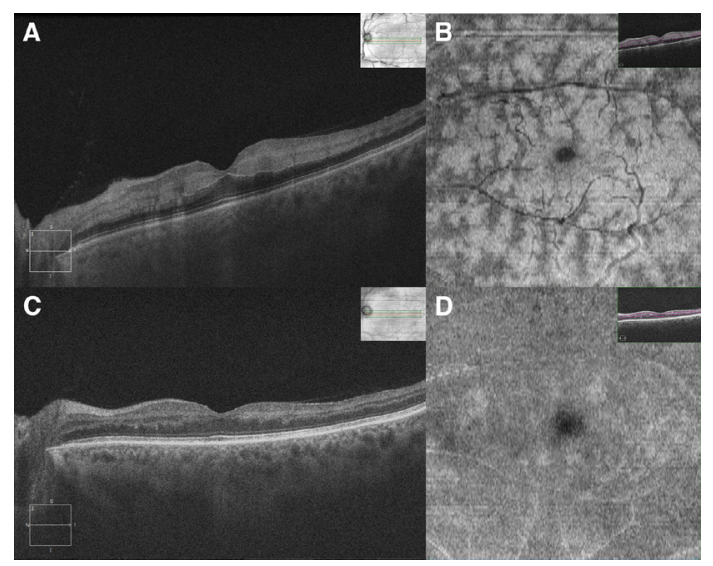

Figure 2 Spectral-domain optical coherence tomography of the macula of the left eye at the time of diagnosis and at 2 months follow-up. The conventional horizontal B-scan (A) demonstrates multifocal hyperreflective plaque-like lesions at the level of the inner nuclear layer (INL), consistent with paracentral acute middle maculopathy (PAMM). The en face visualisation through the mid-retina (B) shows a fern-like pattern of perivenular hyperreflectivity. A follow-up horizontal B-scan (C) demonstrates attenuation of the INL in the area of the previously hyperreflective PAMM lesions. The en face visualisation through the mid-retina (D) shows significant narrowing of the PAMM lesions. 
haemorrhage and venous engorgement, as seen in our case. Almost $50 \%$ of non-ischaemic CRVOs have only mild retinal haemorrhages and up to $20 \%$ do not show any signs of venous engorgement. ${ }^{6}$ In those cases of perivenular PAMM and minimal fundus changes, incomplete retinal arterial occlusion (RAO) must be considered as a differential diagnosis. In our case, the presence of mild venous tortuosity and retinal haemorrhages made non-ischaemic CRVO more likely than partial central RAO. In RAO, ischaemia to the DCP occurs as a result of primary hypoperfusion from reduced arterial inflow pressure. ${ }^{7}$ Isolated perivenular PAMM can be a first sign of 'the ischaemic cascade' that eventually leads to progressive infarction of the middle and inner retina (completion of the RAO). Distinguishing those two aetiologies (venous vs arterial) carries important systemic implications as RAOs typically require urgent stroke work-ups. Digital ophthalmodynamometry and close serial examinations looking for new fundus findings (eg, new haemorrhages vs cherry-red spot from evolution to complete RAO) might be useful to distinguish RVO from RAO. ${ }^{7}$

Identifying perivenular PAMM can also provide insights into the cause of vision loss in CRVO and may provide clues regarding visual prognosis. The fern-like pattern of PAMM suggests ischaemia from arteriolar insufficiency in the DCP leading to

Learning points

- Paracentral scotomas and specific patterns of central vision loss in patients with suspected central retinal vein occlusions (CRVO) should raise suspicion for concurrent paracentral acute middle maculopathy (PAMM).

- En face optical coherence tomography provides frontal sections of retinal layers that allow clinicians to identify different patterns of PAMM: arteriolar, globular and fern-like.

- Fern-like PAMM can complicate non-ischaemic CRVO and its identification can provide insight into the cause of vision loss in those patients. damage at the level of the OPL/INL. This is further supported by the thinning of the INL on follow-up imaging, accounting for the persistent paracentral scotomas in patients with PAMM complicating non-ischaemic CRVO.

\section{Twitter Fares Antaki @FaresAntaki}

Contributors FA diagnosed and followed the patient. FA and RGC conceived the paper. JYS collected the data. FA, JYS and DM interpreted the multimodal retinal imaging. JYS and DM drafted the initial manuscript. FA prepared the figures. FA and RGC supervised the project. All authors had access to the underlying data which they verified. All authors edited and revised the manuscript before approving its final version.

Funding The authors have not declared a specific grant for this research from any funding agency in the public, commercial or not-for-profit sectors.

Competing interests FA reports grants from Bayer Healthcare Pharmaceuticals, personal fees from SNELL Medical Communication Inc, outside the submitted work.

Patient consent for publication Consent obtained directly from patient.

Provenance and peer review Not commissioned; externally peer reviewed.

Case reports provide a valuable learning resource for the scientific community and can indicate areas of interest for future research. They should not be used in isolation to guide treatment choices or public health policy.

\section{ORCID iD}

Fares Antaki http://orcid.org/0000-0001-6679-7276

\section{REFERENCES}

1 Rahimy E, Sarraf D, Dollin ML, et al. Paracentral acute middle maculopathy in nonischemic central retinal vein occlusion. Am J Ophthalmol 2014;158:372-80. e1.

2 Moura-Coelho N, Gaspar T, Ferreira JT, et al. Paracentral acute middle maculopathyreview of the literature. Graefes Arch Clin Exp Ophthalmol 2020;258:1-14.

3 Riazi-Esfahani H, Khalili Pour E, Fadakar K, et al. Multimodal imaging for paracentral acute maculopathy; the diagnostic role of en face OCT. Int J Retin Vitr 2021;7:1-10.

4 Sridhar J, Shahlaee A, Rahimy E, et al. Optical coherence tomography angiography and en face optical coherence tomography features of paracentral acute middle maculopathy. Am J Ophthalmol 2015;160:1259-68. e2.

5 Garrity ST, Tseng VL, Sarraf D. Paracentral acute middle maculopathy in a perivenular fern-like distribution with en face optical coherence tomography. Retin Cases Brief Rep 2018;12:\$25-8

6 Hayreh SS, Zimmerman MB. Fundus changes in central retinal vein occlusion. Retina 2015;35:29-42

7 Zhao PY, Johnson MW, McDonald HR. Paracentral acute middle maculopathy and the ischemic cascade: towards interventional management. Am J Ophthalmol 2021;131.

Copyright 2021 BMJ Publishing Group. All rights reserved. For permission to reuse any of this content visit

https://www.bmj.com/company/products-services/rights-and-licensing/permissions/

BMJ Case Report Fellows may re-use this article for personal use and teaching without any further permission.

Become a Fellow of BMJ Case Reports today and you can:

- Submit as many cases as you like

- Enjoy fast sympathetic peer review and rapid publication of accepted articles

- Access all the published articles

- Re-use any of the published material for personal use and teaching without further permission

Customer Service

If you have any further queries about your subscription, please contact our customer services team on +44 (0) 2071111105 or via email at support@bmj.com.

Visit casereports.bmj.com for more articles like this and to become a Fellow 